\title{
Small molecules exploiting structural differences within microRNA-2oo precursors family members reverse a type 2 diabetes phenotype
}

Hafeez S. Haniffa, , Xiaohui Liua, , Laurent Knerrb, Malin Lemurellb, Daniel Abegga, Alexander Adibekiana, and Matthew D. Disneya,2

a The Scripps Research Institute, Department of Chemistry, 130 Scripps Way, Jupiter, FL 33458, USA

${ }^{\mathrm{b}}$ Medicinal Chemistry, Research and Early Development Cardiovascular, Renal and Metabolism, BioPharmaceuticals R\&D, AstraZeneca, Gothenburg, Pepparedsleden, 1, SE-431 83 Mölndal, Sweden

${ }^{1}$ These authors contributed equally to this work.

${ }^{2}$ Author to whom correspondence should be addressed

Email: disney@scripps.edu

Classification: Major: Biological Science; Minor: Biochemistry 


\begin{abstract}
MicroRNA families are pervasive in the human transcriptome, but specific targeting of individual members is a challenge because of sequence homology. Many of the secondary structures of the precursors to these miRs (pre-miRs), however, are quite different. Here, we demonstrate both in vitro and in cellulis that design of structure-specific small molecules can inhibit specific miR family members to modulate a disease pathway. In particular, the miR-200 family consists five miRs, miR-200a, -20ob, -200c, -141, and 429, and is associated with Type II Diabetes (T2D). We designed a small molecule that potently and selectively targets pre-miR-200c's structure. The compound reverses a proapoptotic effect in a pancreatic $\beta$-cell model. In contrast, oligonucleotides targeting the RNA's sequence inhibit all family members. Global proteomics analysis further demonstrates selectivity for miR-200c. Collectively, these studies establish that miR200c plays an important role in T2D and that small molecules targeting RNA structure can be an important complement to oligonucleotides targeting sequence.
\end{abstract}




\section{Significance Statement}

The most common way to develop medicines targeting RNA is by using oligonucleotides that target its sequence by using base pairing. Some RNAs, however, have similar sequences and thus are impossible to target selectively by using oligonucleotides. Here, we show that a class of RNAs that have similar sequences emerge

from precursors that have very different structures. Exploiting these structural differences afforded a selective compound. In particular, the selective small molecule targets a member of the microRNA (miR)-200 family, the overexpression of which is linked to diabetes and pancreatic cell death. Selective inhibition of family member miR$200 c$ alleviates pancreatic cell death, and thus the small molecule provides a path to the treatment of diabetes. 
/body

RNA structure influences and controls biology, lending the biomolecule its diverse functions. The ability to target RNA structure with synthetic ligands has been a strategy to dissect and understand RNA biology and to leverage it for the development of therapeutics (1-4). One class of RNAs particularly amendable to targeting with synthetic ligands is non-coding microRNAs (miRs) (5). These small regulatory RNAs are synthesized as a primary microRNA transcripts (pri-miRs) that are processed in the nucleus by the microprocessor complex Drosha-DGCR8, affording precursor microRNAs (pre-miRs). The pre-miRs are translocated to the cytoplasm and processed by the nuclease Dicer liberating the mature microRNAs (miR) (6). The miRs bind to mRNAs with sequence complementarity in the 3' untranslated region (3' UTR), suppressing translation (7).

Three approaches have been used to develop miR inhibitors: (i) oligonucleotidebased antagomirs to bind to the mature miRs via base pairing (8); (ii) small molecules that target pri- or pre-miR structures found at functional Dicer or Drosha processing sites, thereby inhibiting biogenesis (9-11); and (iii) small molecules that target miR precursor structures to trigger cleavage of the RNA target (12-14). One of the challenges with targeting some miRs specifically with oligonucleotides is that family members can have the same or similar sequences $(15,16)$. The structures of the precursors to these miRs, however, can be quite different $(15,16)$. The identification of ligands that exploit these differences can thus provide a way to selectively inhibit one miR family member to study its biology and provide leads for precision medicines. Herein, we study the ability to 
differentially affect the biology of miR-200c, a member of the miR-20o family containing miR-200a, -20ob, and -200c, -141, and -429.

Importantly, the miR-200 family contributes to type 2 diabetes (T2D) and, in particular, is causative of $\beta$-cell apoptosis $(17,18)$. Indeed, overexpression of miR-20oc or miR-141 is sufficient to induce $\beta$-cell apoptosis in vivo $(17,18)$. Furthermore, $\beta$-cell apoptosis can be reduced by ablation of the entire miR-20o cluster (18). The miR-200c family is broken down into two subfamilies based on sequence similarity of the seed sequence that forms base pairing interactions with mRNA 3' UTRs: miR-20ob, -200c, and -429 form one sub-family while miR-141 and -200 form the other $(19,20)$. This overlap makes dissecting the contribution of each miR to $\beta$-cell -function and survival challenging.

Herein, we show that oligonucleotide-based approaches that target RNA sequence cannot discriminate broadly against the miR-200 family because of sequence homology. Analysis of the precursors from which the miRs emanate, however, shows broad differences in the RNA structures that could be exploited with structure-specific organic ligands (Figure 1). Indeed, we designed a structure-binding ligand that is a selective inhibitor of pre-miR-200c biogenesis. Further, the compound exerts selective and potent effects on the miRnome and the proteome and stimulates beneficial effects on a T2Dassociated phenotype in a $\beta$-cell model. Thus, these studies validate that miR-20oc is a phenotype-driving biomolecule.

\section{Results}

Analysis of the miRNA-2oo family to design structure-specific

ligands. MiRNA families are a class of non-coding RNAs that have similar functions, 
however they are not necessarily conserved in their primary sequence nor in the structure of their precursors. The miR-200 family comprises five members that have been linked to T2D, causing $\beta$-cell mass loss by triggering apoptosis and decreasing insulin production (18). Thus, inhibitors of these miRs could serve as chemical probes to study their function and potentially as treatments of T2D if properly developed. Across the miR-200 family, miR-200c has been suggested as a driver of $\beta$-cell mass reduction (Figure 1A). Thus, we sought to design ligands to specifically inhibit this target by recognition of the threedimensional structure of pre-miR-200c. By targeting this member of the miR-200 family specifically, we open the way for further investigation into the role of miR-20oc in $\beta$-cell mass regulation.

We analyzed the sequences and structures of all miR-20o family members, which are shown in Figure $\mathbf{1 A} \& \mathbf{B}(19,20)$. Not surprisingly, a high degree of sequence homology was observed between the mature $3 p$ miRNAs, ranging from $72 \%$ to $95 \%$, suggesting that sequence-based recognition would not be specific enough to inhibit a singular miR. To test if this is indeed the case, we obtained a phosphorothioate antagomir with locked nucleic acid (LNA) modifications complementary to 21 nucleotides of mature miR-200c-3p from Qiagen (Figure 1B) and studied its effect on the levels of all mature miR-200 family members by RT-qPCR in a validated cellular model of T2D, the mouse pancreatic $\beta$ cell line MIN6 forced to express pre-miR-200c (18). [Note: the mouse and human miR-200 family are highly conserved both from a sequence and structural perspective (Figure S1). Sequence similarity ranges from $54-98 \%$. The antagomir oligonucleotide is $67-100 \%$ complementary to the family members, but fully complementary to miR-20oc-3p (Figure S1).] Not unexpectedly, the oligonucleotide 
could not discriminate between the family members in the mouse pancreatic $\beta$ cell line MIN6 at any concentration tested (0.1 - $100 \mathrm{nM}$; Figure 1C), even with forced expression of miR-200c and endogenous levels of the others.

In contrast with the high degree of sequence homology between miR-200 family members, significant differences in the structures of their precursors were observed (Figure 1B; see Figure S1 for a comparison of the structures of mouse and human miRs). This suggests that a structure-targeting ligand may be able discriminate amongst the family members. Our previous studies have shown that binding of small molecules to functional sites in miRs, i.e., Drosha and Dicer processing sites $(7,21)$, can impede miRNA biogenesis, de-repress downstream proteins, and rescue disease phenotype; small molecules that bind to other sites in the miRNA are inactive $(5,22)$. Fortuitously, our analysis also revealed that the $1 \times 1$ nucleotide UU Internal loop in pre-miR-20oc's Dicer site is unique across the family and thus is an ideal target site against which to develop a specific ligand. [Note the structural similarity between mouse and human pre-miR-20oc, including the Dicer site and adjacent regions (Figure S1).]

We queried our database of RNA structure-small molecule interactions to identify lead small molecules for the UU internal loop in miR-20oc's Dicer processing site, i.e., our lead identification strategy Inforna $(11,23)$. Inforna identified a promising lead in compound 1 (Figure 2), which bound avidly to this structure with a $\mathrm{K}_{\mathrm{d}}$ of $1.1 \pm 0.08 \mu \mathrm{M}$. The compound had no measurable affinity to an RNA with a single nucleotide change that alters the $1 \times 1$ nucleotide UU internal loop to a base pair (Figure S2). In vitro, $\mathbf{1}$ inhibited Dicer processing of pre-miR-200c with an $\mathrm{IC}_{50}$ of $2.2 \pm 0.5 \mu \mathrm{M}$, with no effect on processing of a control pre-miR-200c RNA in which the binding site for $\mathbf{1}$ was mutated to 
an AU base pair (Figure S3). In MIN6 cells, 1 modestly reduced mature miR-20oc levels $(\sim 35 \%$ knock down at a dose of $20 \mu \mathrm{M})$ but was selective across the miR-20o family (Figure S4). Further, the reduction in mature miR-20oc levels by $\mathbf{1}$ was dose-dependent (Figure S5A); in agreement with its designed mode of action, $\mathbf{1}$ also boosted levels of pre-miR-200c, by $\sim 50 \%$ at a $20 \mu \mathrm{M}$ dose (Figure S5B). As a result of the inhibition of pre-miR-200c biogenesis, 1 de-repressed the miR's downstream mRNA targets $R p s 6 k b 1$ (ribosomal protein S6 kinase $\beta-1$ isoform), Dnajc3 (DnaJ Heat Shock Protein Family (Hsp40) Member C3), Xiap (X-linked inhibitor of apoptosis), and Jazf1 (juxtaposed with another Zinc finger protein 1) (Figure S5C), and inhibited $\beta$-cell apoptosis, a miR-20ocassociated phenotype in T2D (Figure S5D).

\section{Inforna-based optimization of 1 increases in vitro affinity and}

potency. Given that $\mathbf{1}$ binds to the $1 \times 1$ nucleotide $U U$ internal loop at the Dicer site in pre-miR-200c with modest affinity and inhibits pre-miR-200c with good selectivity in pancreatic $\beta$ cells, it provides a promising lead for further optimization. One way to increase affinity, selectivity, and potency is to bind two or more structural motifs simultaneously with a single small molecule. Typically, we have designed modularly assembled compounds that target two adjacent non-canonically paired motifs, such as internal loops that are in close proximity (24). In pre-miR-200c, however, such sites are distant from the binding site of $\mathbf{1}$. Thus, we designed a heterodimer comprising $\mathbf{1}$ and ligand $\mathbf{2}$ that binds a 5'UU/3'AA base pair motif adjacent to the Dicer processing site with high affinity and selectivity (Figure 2) (25).

Synthesis of the heterodimer required modification of $\mathbf{1}$ and $\mathbf{2}$ with functional handles for conjugation. Specifically, $\mathbf{1}$ was functionalized with an alkyne for azide-alkyne 
click chemistry (26), affording $\mathbf{3}$, while $\mathbf{2}$ was functionalized with a butanoic acid linker for subsequent coupling to an amine, affording 4, respectively. Analysis of $\mathbf{3}$ and $\mathbf{4}$ showed that they have similar in vitro and biological activity compared to their respective parent compounds 1 and 2 (Figures S6 - S8). [Note: 2 and 4 did not inhibit Dicer processing of pre-miR-200c in vitro or in MIN6 cells, as expected as it does not bind the Dicer processing site, but did bind pre-miR-200c with modest affinity.] The RNA-binding modules were assembled onto peptoid scaffolds of different lengths $(\mathrm{n}=0,1$, and 2 spacing modules) to identify the one that most closely mimics the distance between premiR-20oc's Dicer site and adjacent AU pairs (Figure 2). The most promising dimer was identified by screening the three dimers for inhibiting apoptosis of MIN6 cells at 0.02 , 0.2, $2 \mu \mathrm{M}$ concentrations. While dimers with $\mathrm{o}$ and 2 spacing modules were inactive, the dimer with one $N$-propyl glycine spacing module, 5, exhibited dose dependent rescue of apoptosis (reduction of Caspase 3/7 activity; Figure S9A \& B). Notably, TGP-20oc was non-toxic to MIN6 cells at these concentrations (Figure S9C). Heretofore, 5 is referred to as TGP-200c (Targapremir-200c) as it selectively and potently targets premiR-20oc vide infra.

We next studied TGP-200c in vitro and compared it with lead compound 1. TGP200c bound pre-miR-200c with a $\mathrm{K}_{\mathrm{d}}$ of $50 \pm 8 \mathrm{nM}, 22$-fold more avidly than $\mathbf{1}$ (Figure S6). Furthermore, no binding was observed when either the $1 \times 1$ nucleotide UU internal loop was mutated to an AU base pair or when the 5'UU/3'AA motif was mutated to GC pairs (Figure S6). Thus, both the internal loop and the adjacent 5'UU/3'AA are required for binding of TGP-200c. TGP-20oc's enhanced affinity translated into enhanced potency in vitro, inhibiting Dicer processing of pre-miR-200c with an $\mathrm{IC}_{50}$ of $\sim 200 \mathrm{nM}$ nM, 10-fold more potent than 1 (Figures S3A \& S1OA). Importantly, TGP-2ooc had 
no inhibitory effect on Dicer processing of a mutant pre-miR-200c in which the binding site for 1 was ablated (Figure S1oB). Collectively the optimized assembled compound has improved binding affinity and potency relative to starting monomer $\mathbf{1}$ and is highly selective.

TGP-2OOc occupies target pre-miR-2oOc in cells to a greater extent than 1. Chemical Cross-Linking and Isolation by Pull-down (Chem-CLIP) is a target validation method that can also be used to study target occupancy and selectivity in vitro and in cells (27). To study occupancy of pre-miR-200c, a Chem-CLIP probe was designed and synthesized. The probe, $\mathbf{6}$, was constructed by using the alkyne functional group in $\mathbf{3}$ to attach a chlorambucil (CA) cross-linking module and a biotin module that allows for purification of cross-linked material. In MIN6 cells transfected with WT pre-miR-20oc, probe 6 cross-linked with pre-miR-200c dose dependently (Figure 3B). Importantly, 6 did not bind a pre-miR-20oc mutant in which the UU loop binding site for $\mathbf{1}$ was mutated to AU base pair in MIN6 cells, as expected due to its lack of binding affinity (Figures 3B \& S6B).

To assess the relative occupancy of monomer $\mathbf{1}$ and TGP-200c, we used Competitive (C-)Chem-CLIP (5). In this experiment, a constant concentration of the Chem-CLIP probe $6(200 \mathrm{nM}$ ) was added to cells along with increasing doses of unreactive 1 or TGP-200c (20 nM to 20,000 nM), which compete off the reaction of 6 with pre-miR-200c. Both compound 1 and TGP-200c inhibited cross-linking and hence pull down of pre-miR-200x by the Chem-CLIP probe. As expected, TGP-2ooc was a 10fold more effective competitor than $\mathbf{1}$ (Figure $\mathbf{3 C}$ ). All together, in vitro binding analyses, inhibition of Dicer processing in vitro, and cellular target occupancy studies 
suggest that the lead optimized compound more potently targets pre-miR-20oc than starting compound $\mathbf{1}$.

TGP-2OOc potently inhibits miR-2ooc biogenesis selectively across

the miRnome. We first measured TGP-200c's ability to inhibit the miR's biogenesis by measuring its effect on mature miR levels, which should decrease, and pre-miR levels, which should increase. A dose-dependent (0.02, 0.2, $2 \mu \mathrm{M})$ decrease in mature miR-200c levels was indeed observed with an $\mathrm{IC}_{50}$ of $\sim 200 \mathrm{nM}, \sim 300$-fold more potently than $\mathbf{1}$ (Figures $4 \mathbf{A} \& \mathbf{S} 5 \mathbf{A}$ ). Importantly, even at the high dose of $2 \mu \mathrm{M}$, TGP-2ooc had no effect on the other members of the miR-200 family (Figure 4B), in contrast to the sequence-based recognition of the oligonucleotide antagomir (Figure 1C). Consistent with its designed mode of action, TGP-200c increased pre-miR-200c levels (Figure 4C). To assess the selectivity of TGP-200c for inhibiting miR-20oc biogenesis, we studied its effects across all 193 miRs that are detectable in MIN6 cells by RT-qPCR. Indeed, only miR-200c levels were affected significantly, further demonstrating that the compound is potent and selective (Figure 4D).

As mentioned above, in T2D miR-200c acts by repressing the expression of XIAP, RPS6KB1, DNAJC3, and $J A Z F 1$ by binding to their 3' UTRs (Figure 1) (18). Thus, we measured TGP-200c's ability to stimulate their expression levels by RT-qPCR. At a 2 $\mu \mathrm{M}$ dose, TGP-200c increased levels of each mRNA (Figure 5A). Robust antibodies are available for DNAJC3 and RPS6KB1, and thus we measured changes in their protein levels upon treatment with $2 \mu \mathrm{M}$ of TGP-200c by Western blotting. Indeed, we observed an increase in the levels of both proteins by $\sim 40 \%$, similar to the antagomir directed at miR-20Oc (Figure 5B). 
In T2D, aberrant expression of miR-200c triggers apoptosis (Figure $5 \mathbf{C}$ ), an effect that can be directly traced to decreased levels of Xiap and Rps6KB1 (Figure 1). Derepression of these proteins by TGP-200 suggests that the compound may rescue this T2D phenotype. As assessed by Caspase 3/7 activity, TGP-2ooc inhibited apoptosis on MIN6 cells transfected with pre-miR-200c dose dependently (Figure 5D). Thus, TGP200c deactivates a T2D circuit at the level of the transcriptome, proteome, and phenotype.

To confirm the binding site dependence on the bioactivity of TGP-200c, we studied its effect on MIN6 cells expressing the mutant pre-miR-200c (mut-miR-200c) in which the compound's binding site is ablated; the mutant is processed by Dicer but is not inhibited by TGP-2ooc (Figure S11). As expected, TGP-200c was unable to inhibit the biogenesis of mut-miR-200c, de-repress Xiap, Rps6kb1, Dnajc3 and Jazf1 mRNA levels, or inhibit apoptosis, each in contrast to the LNA antagomir (Figure S11). These studies demonstrate that TGP-200c exerts its specific effects by binding the Dicer site in pre-miR-200c and inhibiting its biogenesis.

TGP-2Ooc exerts selective effects on proteome globally. We next studied the selectivity of TGP-200 on the entire proteome, dosing MIN6 cells transfected with pre-miR-200c with $2 \mu \mathrm{M}$ compound. Only 13 of the 4000 detectable proteins were affected, using a false discovery rate (FDR) of $1 \%$. Of these 13 proteins, seven are encoded by mRNAs that are direct targets of miR-200c/20ob/429 which form one of two subfamilies within the miR-200 family (Figure 6A). In contrast, no significant effects are found on proteins whose mRNAs are targets for the miR-200a/miR-141 subfamily (Figures 6A \& 6B). 
Pathway analysis revealed that various T2D-related pathways are modulated by TGP-200c treatment, including insulin signaling and metabolism (Figure 6C). Interestingly, we see an upregulation of proteins that negatively regulate cell death such as AKT2 (28) among others, which is also expected given the anti-apoptotic phenotype of TGP-200c. These observations also support our hypothesis that TGP-200c represses pro-cell death factors and promotes $\beta$-cell survival in specific ways. Collectively, these proteome-wide studies and pathway analyses further bolster the assertion that TGP200c is selective small molecule.

\section{Discussion}

The most common way to target RNAs is by the sequence-based recognition of oligonucleotides (4). RNA 3D-structure, however, directly influence and in some cases control biology (29). Thus, targeting RNA structures with small molecules to perturb their function is a route to affect and study RNA biology (3). There is the perception that human RNAs do not generally fold into well-defined structures. Coupled with the notion that RNA cannot form diverse structures as it is only built on four nucleic acid building blocks, RNA has been mostly thought of as "undruggable" or "unligandable". A variety of studies have challenged this assertion, including those presented herein. Evidently, RNA is indeed a viable small molecule drug target and targeting RNA structures in cells can be done rationally and predictably to provide potent and selective small molecules across a variety of indications (27), now including T2D.

Oligonucleotides are thought to be selective for fully complementary targets, however they can have off-targets (30). These off-targets include RNAs that are fully complementary and those that contain mismatches (30-32). Other factors that affect 
selectivity include the expression levels of the on- and the off-targets, with the more highly expressed target more likely to be occupied and affected (32).

Previously, we have shown that for some RNA targets, structure-specific recognition can be advantageous in disease-affected cells and animal models over sequence-specific recognition (33-36). For example, r(CUG) repeat expansions that cause myotonic dystrophy type 1 form a robustly folded structure that can be targeted selectively with structure-binding small molecules over transcripts with short, unstructured stretches of $\mathrm{r}(\mathrm{CUG})$ repeats (37). In contrast, oligonucleotides complementary to the repeats are unable to distinguish between repeats of length that cause disease and those that do not. In line with those studies, herein we have shown that miR family members sharing sequence homology cannot be selectively inhibited with an oligonucleotide but the structures of their precursors can be distinguished by small molecules, resulting in a selective inhibitor of a single miR's biogenesis as demonstrated by miRnome- and proteome-wide studies. That is, the small molecule exploits a structure unique to premiR-200c amongst its family members, and it exerts specific effects on the transcriptome, proteome, and on phenotype. This selective probe molecule is further believed to be a useful tool to disentangle the biological importance of the individual members in the miR200 family beyond $\beta$-cell apotosis. The miR-200 family is one of the most well studied families within cancer (38), and miR-200c was recently reported to have a relevance in podocyte dysfunction in kidney disease (39).

Interestingly, our designed inhibitor comprises an internal loop-targeting small molecules and a base pair-targeting small molecule. There are limited examples of using such a strategy to target RNA as most dimeric molecules target two non-canonically paired structures (40). There could be a perception that adding a base-pair targeting 
module would negatively impact selectivity, but the variety of studies shown here with TGP-200c demonstrate that this is not necessarily the case. There are likely many more applications of such an approach, which could provide an efficient route to ligands that target RNA in which the linker size and hence molecular weight of the molecule could be reduced.

More broadly, T2D affects a large segment of the world's population and there are very few therapeutic approaches (41). The ability to provide a lead compound that targets an ncRNA potently and selectively to abrogate a pro-apoptotic pathway suggests that these compounds could have utility as leads for further preclinical development. The data presented herein further cement that RNA is indeed targetable with small molecule broadly and across disease indications.

\section{Methods}

General Methods. All RT-qPCR experiments were completed on an Applied Biosystems QS5 384-well qPCR system. Gene expression was measured using Power Sybr Green Master mix (Life Technologies) for all RT-qPCR experiments. Gels with radioactive signal were imaged with a Typhoon FLA9500 (GE Healthcare Life Sciences), and images were processed using BioRad's QuantityOne. RNA oligonucleotides used for binding assays were obtained from Dharmacon and deprotected according the manufacturer's protocol. All RNAs were desalted on PD-10 sephadex columns (GE Healthcare Life Sciences) per the manufacturer's protocol and quantified by UV/Vis on a DU80o UV-Vis spectrophotometer at $90{ }^{\circ} \mathrm{C}$. 
Tissue Culture. MIN6 mouse pancreatic $\beta$ cells (Co018008) were obtained from Addex Bio technologies and checked for mycoplasma contamination every 6 months by PCR assay (Promokine). Cells were maintained in growth medium: optimized DMEM from Addex Bio (catalog number Cooo3-02) supplemented with 20\% (v/v) FBS (Sigma), 1× Penicillin/Streptomycin (Corning), and $35 \mathrm{mM}$ of $\beta$-mercaptoethanol (Sigma). Growth medium was passed through a $0.2 \mu \mathrm{m}$ sterile filter before use. All cells are cultured at 37 ${ }^{\circ} \mathrm{C}$ in $5 \% \mathrm{CO}_{2}$.

PCR amplification and transcription of DNA templates. DNA templates for premiR-200c and the mutant pre-miR-200c were PCR amplified using a forward primer that also encodes for a T7 RNA polymerase promoter and the corresponding a reverse primer (see Table S1). PCR amplification was carried out in $300 \mu \mathrm{L}$ of $1 \times$ PCR buffer (10 mM Tris-HCl, pH 9.0, 50 mM KCl, and 0.1\% (v/v) Triton X-100), $4.25 \mathrm{mM} \mathrm{MgCl}_{2}, 0.33 \mathrm{mM}$ dNTPs, $2 \mu \mathrm{M}$ of each primer, and $1.5 \mu \mathrm{L}$ of Taq DNA polymerase. Amplification was carried out by completing 30 cycles at $95{ }^{\circ} \mathrm{C}$ for $30 \mathrm{~s}, 50{ }^{\circ} \mathrm{C}$ for $30 \mathrm{~s}$, and $72{ }^{\circ} \mathrm{C}$ for $60 \mathrm{~s}$. Amplification was confirmed by a 3\% (w/v) agarose gel stained with ethidium bromide.

In vitro transcription of the DNA templates was completed as reported previously (42). Briefly, $300 \mu \mathrm{L}$ of the PCR product was incubated in $1 \mathrm{~mL}$ of $1 \times$ Transcription buffer (40 mM Tris-HCl, pH 8.0, 1 mM spermidine, $10 \mathrm{mM}$ DTT, and 0.001\% (v/v) Triton X100) containing $2.5 \mathrm{mM}$ of each $\mathrm{rNTP}, 15 \mathrm{mM} \mathrm{MgCl}_{2}$, and $20 \mu \mathrm{L}$ of $20 \mathrm{mg} / \mathrm{mL} \mathrm{T} 7 \mathrm{RNA}$ polymerase overnight at $37^{\circ} \mathrm{C}$. After transcription, 1 unit of RNase-free DNase I (Promega) was added to the reaction, which was incubated at $37^{\circ} \mathrm{C}$ for an additional $1 \mathrm{~h}$. The RNA was purified on a denaturing $15 \%$ polyacrylamide gel and extracted as previously described (42). RNAs were quantified by using its absorbance at $260 \mathrm{~nm}$ at 90 
${ }^{\circ} \mathrm{C}$ and the respective extinction coefficient determined from nearest neighbor parameters (43) and the HyTher server (44).

Inhibition of Dicer processing of pre-miR-2ooc in vitro. Pre-miRs were radioactively labeled on the 5' end and purified as previously described (42). The labeled RNA was folded in $1 \times$ Dicer Reaction Buffer (Genlantis) by heating at $95{ }^{\circ} \mathrm{C}$ for $30 \mathrm{~s}$ and cooling slowly to room temperature. The samples were then supplemented with $1 \mathrm{mM}$ ATP and $2.5 \mathrm{mM} \mathrm{MgCl}_{2}$, followed by addition of 1 or TGP-200c (25 to $0.78 \mu \mathrm{M}$ ). The samples were incubated at room temp for $30 \mathrm{~min}$ followed by addition of recombinant Dicer (BPS-Bioscience) to a final concentration of $0.017 \mathrm{mg} / \mathrm{mL}$. The samples were incubated for $1 \mathrm{~h}$ at $37^{\circ} \mathrm{C}$, and then the reaction was quenched by addition of an equal volume of $2 \times$ Loading Buffer (89 mM Tris-Borate, $\mathrm{pH}$ 8.3, 200 mM EDTA, 7M urea, 1 $\mathrm{mg} / \mathrm{mL}$ bromophenol blue/xylene cyanole).

A T1 ladder (cleaves $G$ residues) was generated by incubating 1 pmol of radioactively labeled RNA in $1 \times$ RNA Sequencing Buffer (20 mM sodium citrate, pH 5.0, $1 \mathrm{mM}$ EDTA, and $7 \mathrm{M}$ urea) at $95{ }^{\circ} \mathrm{C}$ for $30 \mathrm{~s}$ followed by slow cooling to room temperature. RNase T1 was then added to a final concentration of $3 \mathrm{U} / \mu \mathrm{L}$, and the samples were incubated at room temp for $30 \mathrm{~min}$. An RNA hydrolysis ladder was created by incubating 1 pmol of radioactively labeled RNA in $1 \times$ RNA Hydrolysis Buffer (50 mM $\mathrm{NaHCO}_{3}, \mathrm{pH}$ 9.4, and $1 \mathrm{mM}$ EDTA) at $95{ }^{\circ} \mathrm{C}$ for 3 min followed by snap cooling on ice. An equal volume of $2 \times$ Loading Buffer was added to each sample.

The cleavage products were resolved on a $0.7 \mathrm{~mm}$ thick, denaturing $15 \%$ polyacrylamide gel and imaged using a Typhoon FLA 9500 by autoradiography. 
Microscale thermophoresis binding. The binding affinities of $\mathbf{1}, \mathbf{3}, \mathbf{4}$, and TGP200c were measured by microscale thermophoresis (MST). Measurements were taken using standard capillaries (Nano Temper) on a Monolith Nt. 115 system (Nano Temper). Samples were prepared with as follows: $20 \mathrm{nM}$ of RNA was folded in $2 \times$ Binding Buffer (16 $\mathrm{mM} \mathrm{Na}_{2} \mathrm{PO}_{4}, \mathrm{pH} 7.0$, and $370 \mathrm{mM} \mathrm{NaCl}$ ) by heating at $65^{\circ} \mathrm{C}$ for 20 min followed by slow cooling to room temperature on the benchtop. Once cooled, bovine serum albumin (BSA) was added to a concentration of $80 \mu \mathrm{g} / \mathrm{mL}$. Compounds were diluted to $100 \mu \mathrm{M}$ and then serial diluted 1:2 in water. An equal volume of folded RNA has added to each concentration of compound in a total volume of $20 \mu \mathrm{L}$. The samples were incubated at room temp for $15 \mathrm{~min}$ before loading into the capillaries. Instrument settings were as follows: LED power 15\%, MST power 40\%, fluorescence detection before $5 \mathrm{~s}$, MST power on $30 \mathrm{~s}$, fluorescence detection after MST off $5 \mathrm{~s}$, delay before next scan $25 \mathrm{~s}$. Each run was repeated in quadruplicate, and the results averaged. All data were fit according to equation 1 below used previously(45):

$$
I=I_{\mathrm{o}}+0.5 \Delta \epsilon\left(\left([F L]_{\mathrm{o}}+[R N A]_{\mathrm{o}}+K_{t}\right)-\left(\left([F L]_{\mathrm{o}}+[R N A]_{\mathrm{o}}+K_{t}\right)^{2}-4[F L]_{\mathrm{o}}[R N A]_{\mathrm{o}}\right)^{0.5)} \quad(\text { eq.1) }\right.
$$

where $I$ and $I_{0}$ are the observed fluorescence and initial fluorescence intensity in the presence and absence of RNA, $\Delta \varepsilon$ is the difference between the fluorescence intensity in the absence and presence of infinite RNA concentration, $[\mathrm{FL}]_{\mathrm{o}}$ and $[\mathrm{RNA}]_{\mathrm{o}}$ are respectively the concentrations of the small molecule and $\mathrm{RNA}$, and $\mathrm{K}_{\mathrm{t}}$ is the dissociation constant.

Transfection of MIN6 cells. MIN6 cells were grown to $\sim 50 \%$ confluency in $100 \mathrm{~mm}$ dishes and then batch transfected with $1 \mu \mathrm{g}$ of wild type pre-miR-20oc (MmiR-3304- 
MRo4, Genecopoeia) or mutant pre-miR-200c (Synthesized by Genscript) using Lipofectamine 3000 (Life Technologies) per the manufacturer's protocol. Cells were also mock transfected by using the same quantities of reagents for plasmid transfection without the addition of plasmid.

\section{Measuring pre- and mature miR-2ooc and mRNA levels by RT-qPCR}

analysis. After transfection, cells were allowed to recover for $8 \mathrm{~h}$ in complete growth medium before splitting into 12-well plates (100,000 cells/well). After adhering for $12 \mathrm{~h}$, the cells were treated with 1 or TGP-200c in dose response, vehicle (DMSO), LNA-200c, or LNA-Scramble for $60 \mathrm{~h}$. Total RNA was extracted using the Zymo Quick-RNA Miniprep Kit per the manufacturer's protocol, including DNase treatment. Reverse ttranscription (RT) for miRs was completed with $300 \mathrm{ng}$ of total RNA using a miScript II RT Kit (Qiagen) per the manufacturer's protocol. RT of pre-miR-20oc and mRNAs was completed using QScript Kit (Quanta Bio) with 1000 ng of total RNA per the manufacturer's protocol. RT-qPCR was completed using a QS5 384-well PCR system (Applied Biosystems) using Power Sybr Green Master Mix and the manufacturer's Comparative $C_{t}$ with melt standard method, adjusted for a sample volume of $10 \mu \mathrm{L}$. All data were analyzed using the $\Delta \Delta \mathrm{C}_{\mathrm{t}}$ method previously described (46).

Immunoblotting of RPS6KB1 and DNAJC3. Transfected MIN6 cells were seeded into 6-well plates at 200,000 cells/well and allowed to adhere for $12 \mathrm{~h}$. After adhering, cells were treated with 1, TGP-200c, LNA-200c, LNA-Scramble, or vehicle for $96 \mathrm{~h}$. After $60 \mathrm{~h}$, the medium was replaced with fresh medium containing compound. After 96 h, the cells were washed with $1 \times$ DPBS and then trypsinized and pelleted. The pellets were 
washed once with $1 \times$ DPBS before lysis in M-PER buffer (Thermo Fisher) supplemented with $1 \times$ protease inhibitor cocktail (Roche). Samples were subjected to a freeze-thaw cycle (-80 to room temperature) to ensure complete lysis. Total protein samples were quantified using a Pierce Micro BCA Protein Assay Kit (Fisher Scientific) per the manufacturer's protocol. Approximately $20 \mu \mathrm{g}$ of total protein weas resolved on a $10 \%$ SDS-polyacrylamide gel and then transferred to a PVDF membrane. The membrane was blocked in $5 \%(\mathrm{w} / \mathrm{v})$ milk in $1 \times$ TBST $(1 \times$ TBS, $\mathrm{pH} 7$ supplemented with $0.1 \%(\mathrm{v} / \mathrm{v})$ Tween 20) for $30 \mathrm{~min}$ at room temperature. The membrane was then incubated with $1 \times$ TBST containing 5\% milk and either anti-RPS6KB1 (Cell Signaling Technology, 2940S) or antiDNAJC3 (Cell Signaling Technology, 2708s) primary antibody (1: 2000) at $4{ }^{\circ} \mathrm{C}$ overnight. The membrane was washed $1 \times$ TBST three times for $10 \mathrm{~min}$ each followed by incubation with anti-rabbit IgG horseradish-peroxidase secondary antibody conjugate (1:300o dilution; Cell Signaling Technology, 7074S) in $1 \times$ TBS for 2 h. After washing with $1 \times$ TBST $(3 \times 10 \mathrm{~min})$, protein expression was detected using SuperSignal West Pico Chemiluminescent Substrate (Thermo Fisher) according to the manufacturer's protocol.

The membrane was then stripped by incubating with $1 \times$ Stripping Buffer $(200 \mathrm{mM}$ glycine and $0.1 \% \mathrm{SDS}, \mathrm{pH} 2.2$ ) for $1 \mathrm{~h}$ at room temperature. The membrane was washed with $1 \times$ TBS for 20 min and then incubated with anti- $\beta$-actin primary antibody (1:10000 dilution; Cell Signaling Technology, 3700S) in $1 \times$ TBST with 5\% milk for $2 \mathrm{~h}$ at room temperature. The membrane was then washed with $1 \times$ TBST $(3 \times 10 \mathrm{~min})$ and incubated with anti-mouse IgG horseradish-peroxidase secondary antibody conjugate (1:1000o dilution; Cell Signaling Technology, 7076S) in $1 \times$ TBST for $1 \mathrm{~h}$ at room temperature. After washing with $1 \times$ TBST $(3 \times 10 \mathrm{~min}), \beta$-actin expression was detected as described above. 
ImageJ software was used to quantify the intensities of the protein bands in all Western blots.

Cell viability and Caspase 3/7 Assay. Transfected MIN6 cells (15,00o cells per well) were seeded into 96-well white, clear bottom tissue culture plates (VWR: 89091-014) in $90 \mu \mathrm{L}$ of growth medium and allowed to adhere for $12 \mathrm{~h}$. The cells were then dosed with the compound or oligonucleotide of interest or vehicle and incubated for $60 \mathrm{~h}$. Cell viability was measured using CellTiter Fluor (Promega) according to the manufacturer's protocol, and fluorescence was measured using a Molecular Devices M5 plate reader.

To measure Caspase 3/7 activity, after the treatment period, $100 \mu \mathrm{L} /$ well of Caspase 3/7 reagent (Promega) was added per the manufacturer's protocol. Luminescence was measured on a Molecular Devices M5 plate reader, set to an integration time of $500 \mathrm{~ms}$. Data were processed by first normalizing cell viability to vehicle controls and then normalizing the resulting data to Caspase 3/7 luminescence.

\section{Cellular Chem-CLIP and Competitive-Chem-CLIP of pre-miR-2ooc. MIN6} cells transiently expressing pre-miR-200c were treated with 1-CA-Biotin (10, 1, and 0.1 $\mu \mathrm{M}$ ) for $48 \mathrm{~h}$ in a $60 \mathrm{~mm}$ dish. After $48 \mathrm{~h}$, total RNA was extracted using the miRNeasy Mini Kit (Qiagen) per the manufacturer's protocol, with DNase I treatment. Then, $15 \mu \mathrm{g}$ of total RNA in $500 \mathrm{uL}$ of $1 \times$ DNA buffer $\left(8 \mathrm{mM} \mathrm{Na}_{2} \mathrm{PO}_{4}, \mathrm{pH} 7.0,185 \mathrm{mM} \mathrm{NaCl}\right)$ was incubated with $200 \mu \mathrm{L}$ of a slurry of Dynabeads MyOne Streptavidin C1 (Invitrogen). The samples were incubated with shaking for $5 \mathrm{~h}$ at room temperature. The beads were then captured on a magnetic rack and washed with $1 \times$ Wash Buffer (10 mM Tris-HCl, pH 7.2, $1 \mathrm{mM}$ EDTA, and $4 \mathrm{M} \mathrm{NaCl}$ ) six times and once with nanopure water. The bound RNA 
was then released by incubating the beads with $150 \mu \mathrm{L}$ of $95 \%$ formamide containing 10 mM EDTA at $95{ }^{\circ} \mathrm{C}$ for 30 min. The released RNA was then cleaned up by adding 2 volumes of RNA Lysis Buffer (Zymo Research) and one volume of 100\% ethanol. The sample was loaded onto Quick RNA Miniprep columns (Zymo Research) and then proceeding according to the manufacturer's protocol. RT-qPCR was carried out on premiR-200c as described above using 5 ng of input cDNA from before and after pull-down. Enrichment was calculated as previously described (22). C-Chem-CLIP was completed by pre-treating MIN6 cells with $\mathbf{1}(0.2,2,20 \mu \mathrm{M})$ or TGP-200c $(0.02,0.2,2 \mu \mathrm{M})$ for 15 min followed by addition of $0.2 \mu \mathrm{M}$ 1-CA-Biotin for $48 \mathrm{~h}$. Sample processing and analysis were carried out as described above.

Global proteomics profiling using LC-MS/MS. Cells were harvested and suspended in $1 \times$ PBS and then lysed via sonication. Protein concentration was determined using a Bradford assay (BioRad). Samples (20 $\mu$ g) were denatured with $6 \mathrm{M}$ urea in $50 \mathrm{mM} \mathrm{NH}_{4} \mathrm{HCO}_{3} \mathrm{pH}$, reduced with $10 \mathrm{mM}$ tris(2-carboxyethyl)phosphine hydrochloride (TCEP) for $30 \mathrm{~min}$, and finally alkylated with $25 \mathrm{mM}$ iodoacetamide for 30 min in the dark. Samples were diluted to $2 \mathrm{M}$ urea containing $50 \mathrm{mM} \mathrm{NH}_{4} \mathrm{HCO}_{3} \mathrm{pH} 8$ and digested with trypsin $(1 \mu \mathrm{L}$ of $0.5 \mu \mathrm{g} / \mu \mathrm{L})$ in the presence of $1 \mathrm{mM} \mathrm{CaCl}_{2}$ for $12 \mathrm{~h}$ at 37 ${ }^{\circ} \mathrm{C}$. Samples were acidified with acetic acid to a final concentration of $5 \%$, desalted over a self-packed C18 spin column, and dried. Samples were analyzed by LC-MS/MS (below) and the MS data were processed with MaxQuant (below).

LC-MS/MS analysis. Peptides were resuspended in water with $0.1 \%$ formic acid (FA) and analyzed using EASY-nLC 1200 nano-UHPLC coupled to Q Exactive HF-X Quadrupole-Orbitrap mass spectrometer (Thermo Scientific). The chromatography 
column consisted of a $50 \mathrm{~cm}$ long, $75 \mu \mathrm{m}$ i.d. microcapillary capped by a $5 \mu \mathrm{m}$ tip and packed with ReproSil-Pur 120 C18-AQ 2.4 $\mu \mathrm{m}$ beads (Dr. Maisch GmbH). LC solvents were $0.1 \%$ FA in $\mathrm{H}_{2} \mathrm{O}$ (Buffer A) and 0.1\% FA in 90\% MeCN: $10 \% \mathrm{H}_{2} \mathrm{O}$ (Buffer B). Peptides were eluted into the mass spectrometer at a flow rate of $300 \mathrm{~nL} / \mathrm{min}$ over a $240 \mathrm{~min}$ linear gradient (5-35\% Buffer B) at $65{ }^{\circ} \mathrm{C}$. Data were acquired in data-dependent mode (top-20, NCE 28, $\mathrm{R}=7$ '500) after full MS scan ( $\mathrm{R}=60$ '000, $\mathrm{m} / \mathrm{z} 400-1$ '300 $)$. Dynamic exclusion was set to $10 \mathrm{~s}$, peptide match to prefer, and isotope exclusion was enabled.

MaxQuant analysis. MS data were analyzed with MaxQuant(47) (V1.6.1.0) and searched against the mouse proteome (Uniprot) and a common list of contaminants (included in MaxQuant). The first peptide search tolerance was set at $20 \mathrm{ppm} ; 10 \mathrm{ppm}$ was used for the main peptide search; and fragment mass tolerance was set to 0.02 Da. The false discovery rate (FDR) for peptides, proteins and sites identification was set to 1\%. The minimum peptide length was set to 6 amino acids, and peptide re-quantification, label-free quantification (MaxLFQ), and "match between runs were enabled. The minimal number of peptides per protein was set to two. Methionine oxidation was searched as a variable modification and carbamidomethylation of cysteines was searched as a fixed modification. 


\section{Associated Content}

\section{Supporting Information}

The Supporting Information is available free of charge at DOI: [to be filled in]

The following data can be found in the Supporting information, (i) table of oligonucleotides used in this study; (ii) Supporting Information Figures 1-10; and (iii) synthetic methods and characterization of all compounds.

\section{Author Information}

Corresponding Author:

*E-mail: disney@scripps.edu

\section{ORCIDs:}

Matthew D. Disney: 0ooo-0oo1-8486-1796

Hafeez S. Haniff: oooo-0oo2-5561-5251

Conflict of Interest. M.D.D is a founder and consultant of Expansion Therapeutics; L.K. and M.L. are employees of AstraZeneca.

Author Contributions. M.D.D. designed and directed the study; H.S.H. and X.L. designed and executed experiments; H.S.H conducted all cellular and binding experiments; X.L. synthesized and characterized all compounds used; D.A. and A.A. conducted global proteomics analysis of cellular samples; L.K. and M.L. provided critical intellectual feedback on experimental design.

Data Availability. All raw data associated that support the findings of this study are available from the corresponding author upon reasonable request. 
Acknowledgments. This work was funded by the National Institutes of Health (Ro1

GMo97455 to MDD). We would also like to thank Dr. Jessica L. Childs-Disney, Mr. Christopher Williams, and Dr. Bader Zarrouki (AstraZeneca) for their input and editing during the writing of the manuscript. 
A

$5^{\prime} \mathrm{CC} \mathrm{CU}_{\mathrm{CGUC}}$ UUACCC $^{10} \mathrm{GCAGUGUU} \mathrm{U}_{\mathrm{GGG}} \mathrm{U}_{\mathrm{GC}}^{30} \mathrm{GGU} U$

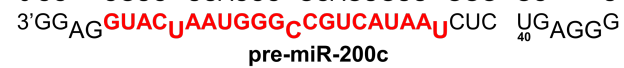

Drosha and Dicer

Processing $\downarrow$

3'AGGUAGUAAUGGGCCGUCAUAAU

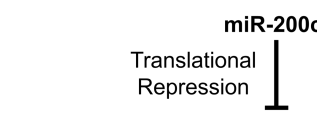

Dnajc3

Repression

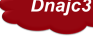

Rpsekb

Jazf1

Roser

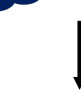

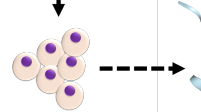

$\beta$ cell mass loss

C

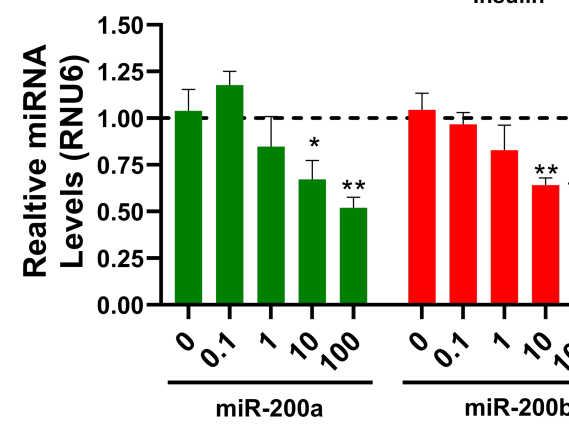

B 5'UggGcauc UUaccGgacagug $\mathrm{C}_{\mathrm{UgGa}} \mathrm{UU}_{\mathrm{UC}} \mathrm{UU}_{\mathrm{G}_{C}}$ 3'ACUUGUAG ${ }_{C} A A U G G U C U G U C A C{ }_{A} A U C U{ }_{C} A_{U U} C$ pre-miR-200a

5'GGAUG $U_{C}$ UUACCAGACA $U_{\text {GUUAGAU }} U_{G G A} U_{G}$ $3^{\prime}{ }^{\prime} C{ }^{\prime} U G C{ }_{C} G$ UAUGGUCUGU ${ }_{C A}$ UAAUCUG UCU ${ }_{A} C$ pre-miR-429

$5^{\prime}$ GCCGU $^{G G C}$ CAUC UUAC ${ } G_{\text {GGCAG }} C_{\text {AUUGGA }} U_{A}$ GUG $_{U}$ 3'CGGCA GUAGUAAUG GUCCGUC ${ }_{A}$ UAAUCU $_{C} U_{A G U C}$ pre-miR-200b 5'GGG $\cup_{\text {CCAUCUU }}$ CCAG $_{\text {GCAGUGUUGG }}$ AU $_{\text {GGUU }}$ GAA $_{G U}$ 3'CCC GGUAGAA ${ }_{A U G G U C}$ UGUCACAAUC $C$ UCGA $_{A G U}$ pre-miR-141

$$
\text { miRNA }
$$

$\operatorname{miR}-200 a-3 p$

$\mathrm{miR}-200 \mathrm{~b}-3 \mathrm{p}$

$\mathrm{miR}-200 \mathrm{c}-3 \mathrm{p}$

$\operatorname{miR}-141-3 p$

miR-429-3p

LNA $-200 C-3 p$
\% Complementarity

5' - TADC

5'-TAATACTGCCTGGTAATGAT $G$ - 95

5' -TAATACTGCCGGGTAATGATGGA 100

5' -TAACACTGTCTGGTAAAGATGG- 86

5'-TAATACTGTCTGGTAAAACC $G \mathbf{T}-\quad 72$

3' - TTATGACGGCCCATTACTACC

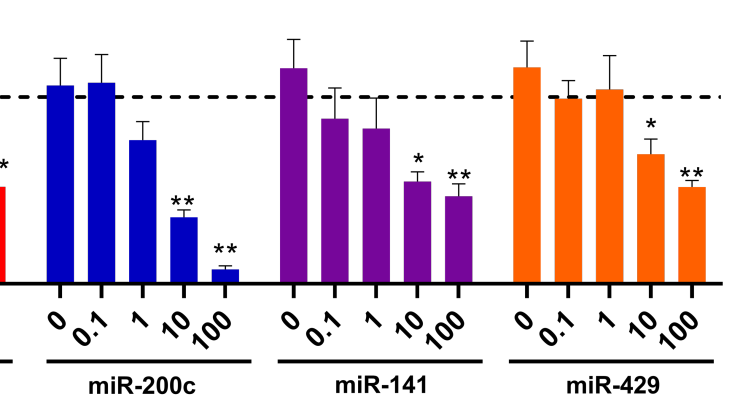

[LNA-200c], nM

Figure 1: Schematic of the miR-20oc pathway, sequence similarity of the miR-20oc

family, and effect of a miR-2ooc-targeting antagomir. A) Precursor miR-20oc is

processed by Dicer to generate mature miR-200c and translationally represses Dnajc3, Rps6kb1, Jazf1, and Xiap. These proteins regulate the activation of apoptosis and thus their downregulation triggers apoptosis of $\beta$-cells and decreases insulin. B) The miR-20o family consists of miR-200a, miR-20ob, miR-200c, miR-141 and miR-429 which each form structurally distinct hairpins but have extensive sequence homology in their mature sequences. C) Effect of LNA200c on all miRNAs in the miR-200 family, which all miR-200 family members as they have $>70 \%$ sequence complementarity to the LNA $(n=4) .{ }^{*}, p<0.05 ;{ }^{* *}, p<0.01$. All p-values were calculated by a two-tailed Student t-test. All data are reported as mean \pm S.E.M. 

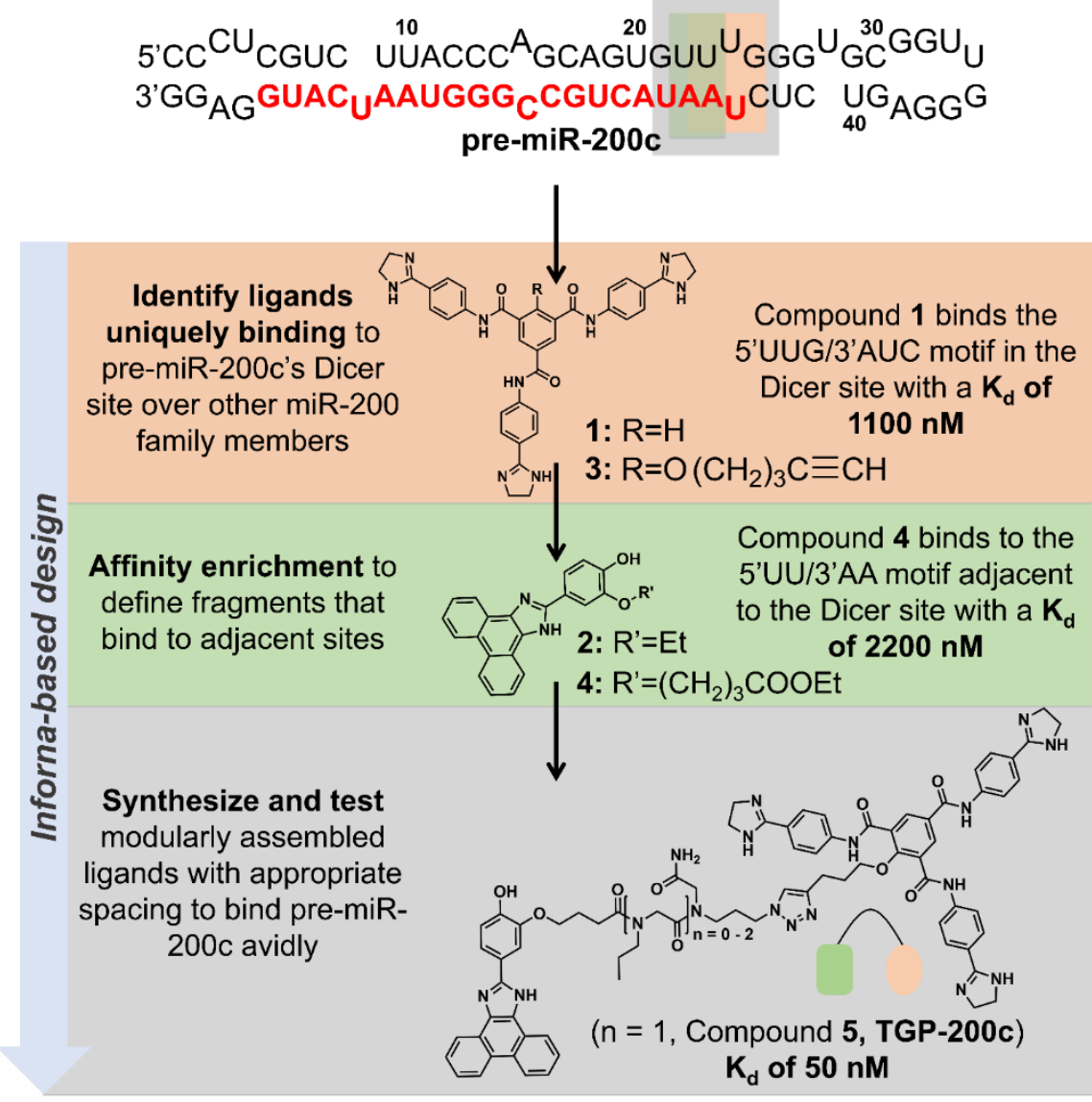

Figure 2: Inforna-based design of small molecules targeting pre-miR-2ooc. The secondary structure of pre-miR-200c was mined via Inforna, identifying compound $\mathbf{1}$ as a binder to the $\mathrm{U} / \mathrm{U}$ loop at the Dicer processing site $\left(\mathrm{K}_{\mathrm{d}}=1100 \mathrm{nM}\right)$. Further analysis identified compound 2 that binds to the adjacent AU base pair $\left(K_{d}=2200 \mathrm{nM}\right)$. Linking these two monomers (via 3 and 4) afforded a more avid binder to the Dicer site of pre-miR-200c, TGP-200c $\left(\mathrm{K}_{\mathrm{d}}=50 \mathrm{nM}\right)$. 
A

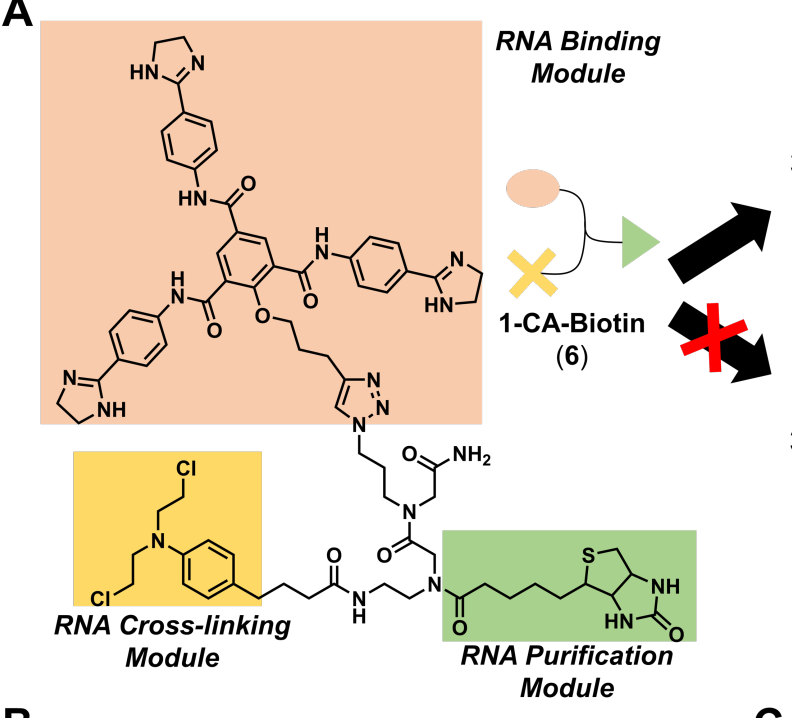

B

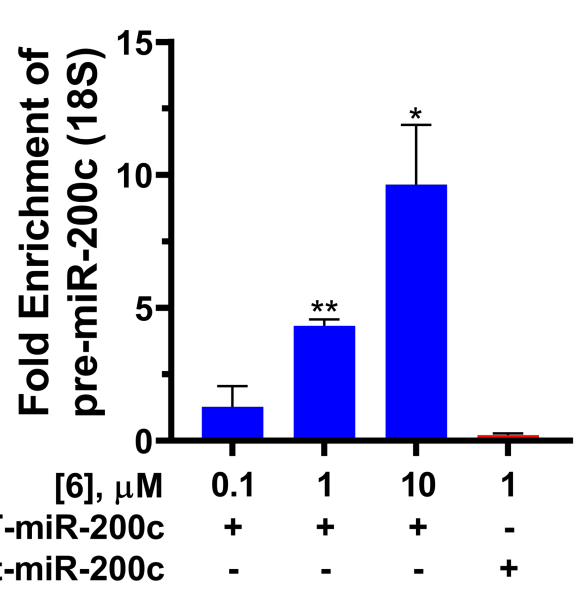

$5^{\prime} C^{C U_{C G U C}}$ UUACCCA ${ }^{A} C A G U G U$ I' ${ }_{3 G G} U_{G C} G^{G U}$

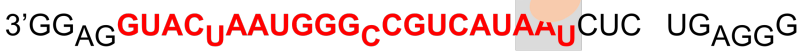

Wild type pre-miR-200c

Binding leading to

reaction and enrichment

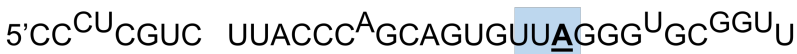

$3^{\prime} G_{A G} G_{A A C}{ }_{U A A U G G}{ }_{C} C G U C A U A A \underline{U} C U C$ UG ${ }_{A G G} G$

Mutant pre-miR-200c

No binding resulting in

no enrichment

C

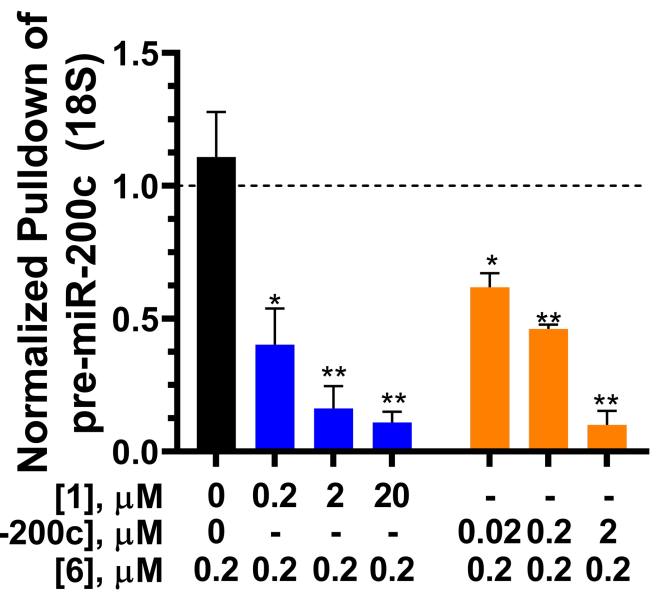

Figure 3: 1 and TGP-20oc selectively engage pre-miR-2ooc in cells. A) Scheme of Chemical-Cross Linking and Isolation by Pulldown (Chem-CLIP) showing that 6 binds and crosslinks to wild type pre-miR-20oc, while not engaging mutant pre-miR-20oc. B) Chem-CLIP studies in MIN6 cells show dose dependent enrichment of pre-miR-20oc by 6 for wild type miR200c (WT-miR-200c), but not mutant miR-200c (Mut miR-200c) in which the binding is ablated $(\mathrm{n}=3)$. C) Competitive-Chem-CLIP shows that $\mathbf{1}$ and TGP-2ooc can compete off the interaction of 6 with pre-miR-200c and, hence cross-linking. Note the 10-fold lower concentrations used for TGP-200 than for $\mathbf{1}(\mathrm{n}=3) .{ }^{*}, \mathrm{p}<0.05 ;{ }^{* *}, \mathrm{p}<0.01$. All $\mathrm{p}$-values were calculated by a two-tailed Student t-test. All data are reported as mean \pm S.E.M. 
A

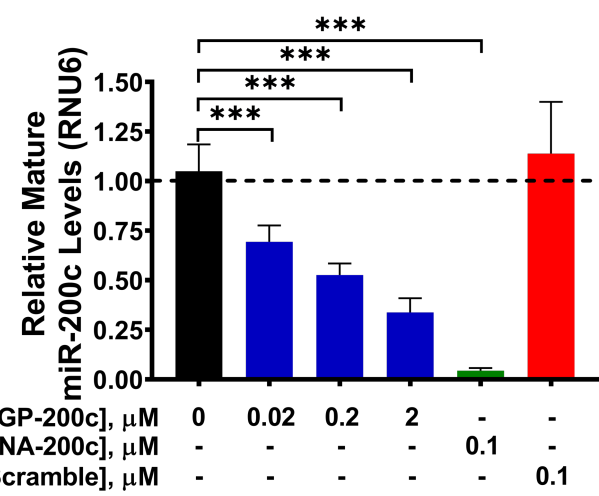

C

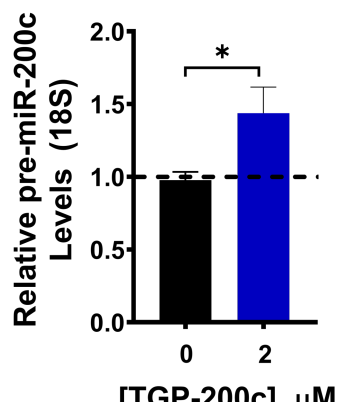

B

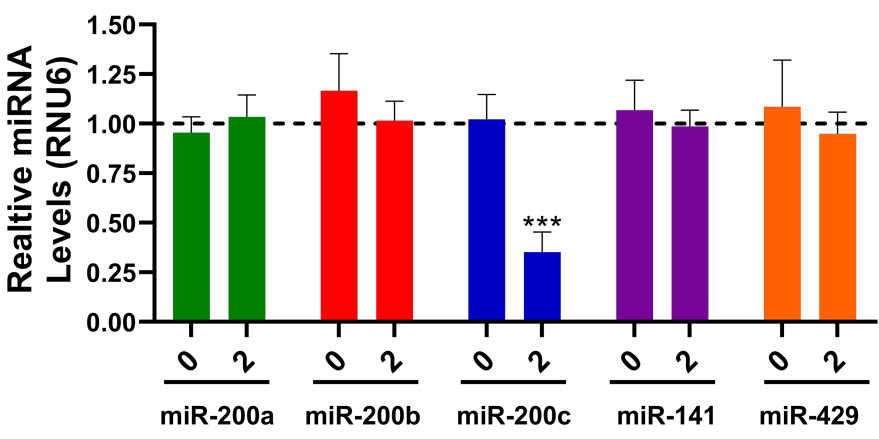

D

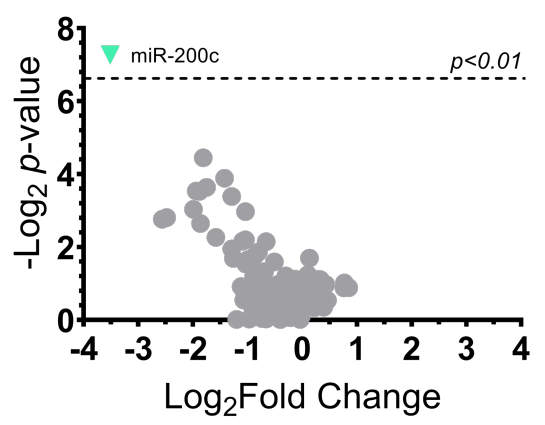

Figure 4: TGP-200c selectively inhibits miR-200c biogenesis in MIN6 cells. A) Effect of TGP-200c on mature miR-200c levels $(n=5)$. B) Effect of TGP-200c on mature levels of all miRs in the miR-200 family $(n=4)$. TGP-200c selectively inhibits miR-200c over all miR-200 family members by recognizing the U/U internal loop unique to pre-miR-20oc. C) Effect of TGP200c on pre-miR-200c levels $(n=4)$. D) Global profiling of all miRs expressed in MIN6 cells shows that only miR-200c levels are significantly downregulated by TGP-200c $(\mathrm{p}<0.01 ; \mathrm{n}=4)$. ${ }^{*}, \mathrm{p}<0.05{ }^{* *}, \mathrm{p}<0.01 .{ }^{* *}, \mathrm{p}<0.001$. All p-values were calculated by a two-tailed Student t-test. All data are reported as mean \pm S.E.M. 
A

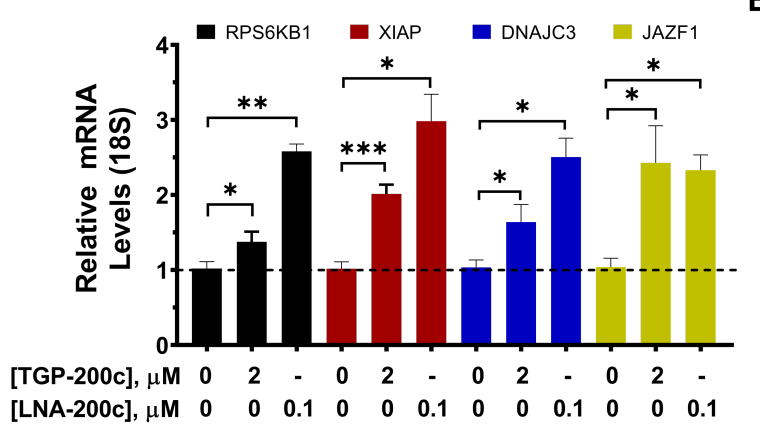

C

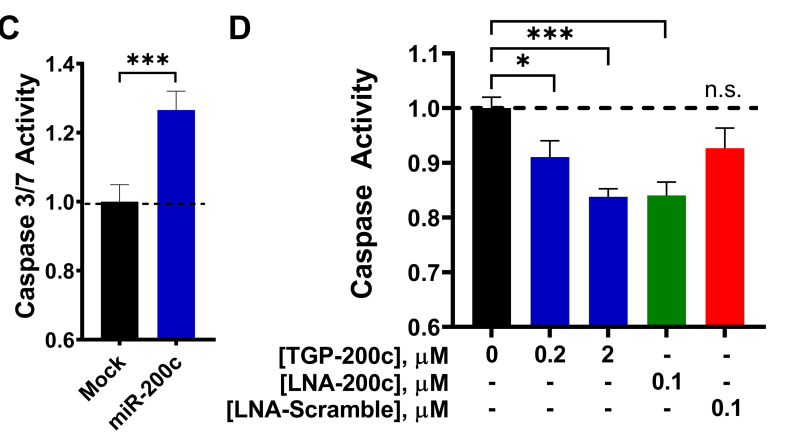

B

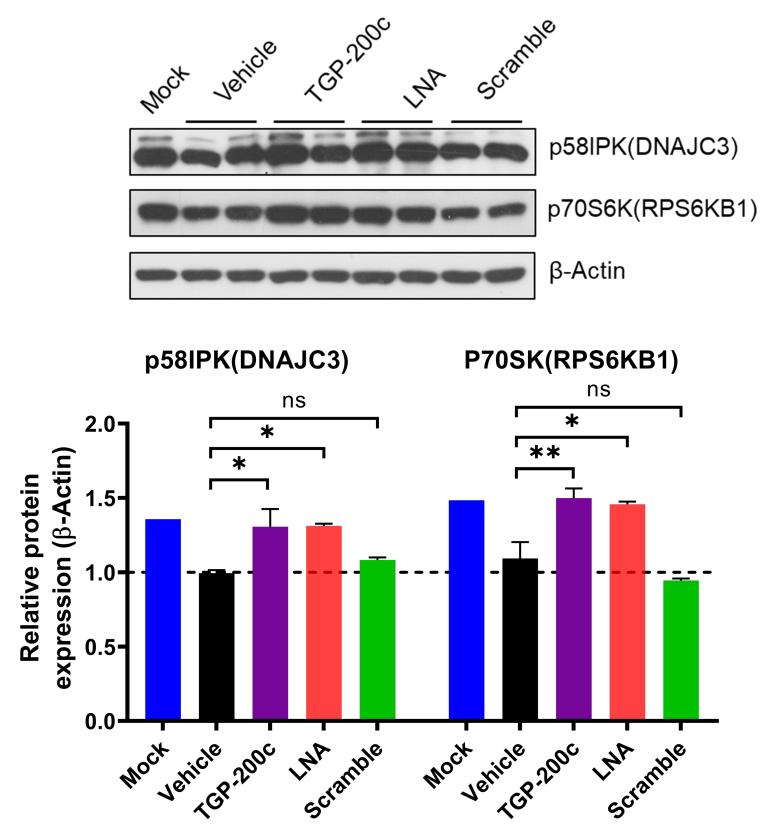

Figure 5: TGP-200c selectively rescues the miR-20oc-mediated phenotype in MIN6

cells. A) Effect of TGP-200c on miR-200c's direct mRNA, Rps6kb1, Dnajc3, Xiap and Jazf1, as determined by RT-qPCR $(n=4)$. B) Effect of TGP-2ooc on p58IPK (DNAJC3) and p70s6K (RPS6KB1) protein levels $(n=1$ for Mock, $n=4$ for Vehicle and TGP-200c, $n=2$ for LNA and scramble). C) Effect of overexpression of pre-miR-200c on the apoptosis of MIN6 pancreatic $\beta$ cells, as determined by measuring Caspase $3 / 7$ activity $(n=16)$. D) Effect of TGP-200c on Caspase $3 / 7$ activity $(\mathrm{n}=8) .{ }^{*}, \mathrm{p}<0.05{ }^{* *}, \mathrm{p}<0.01{ }^{* * *}, \mathrm{p}<0.001$. All $\mathrm{p}$-values were calculated by a two-tailed Student t-test. All data are reported as mean \pm S.E.M. 
A

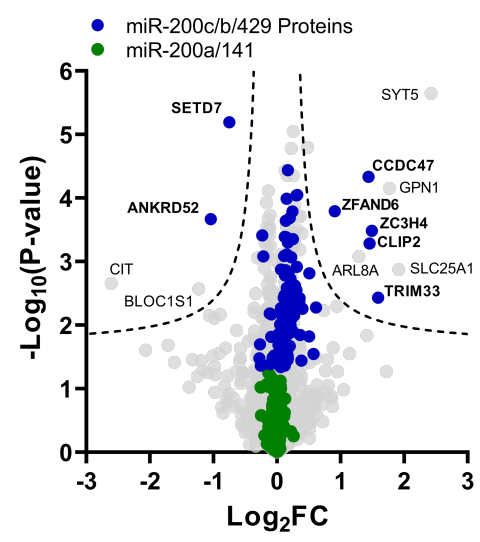

(TGP-200c, $2 \mu \mathrm{M} /$ Vehicle)

B

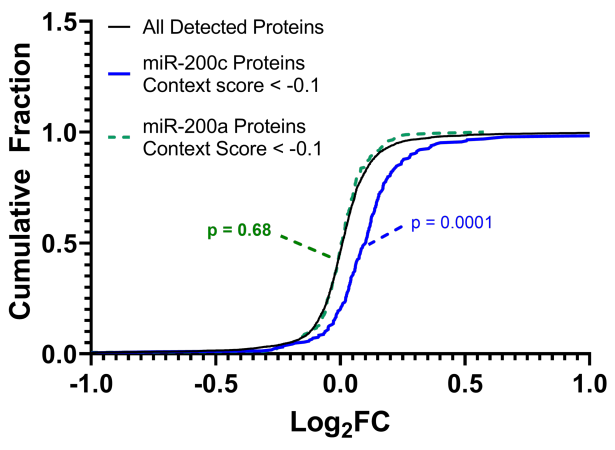

(TGP-200c, $2 \mu \mathrm{M} /$ Vehicle)

C

Regulation of macromolecule metabolism Negative regulation of cell death

Regulation of Metabolism

Insulin signaling

Pathway unclear
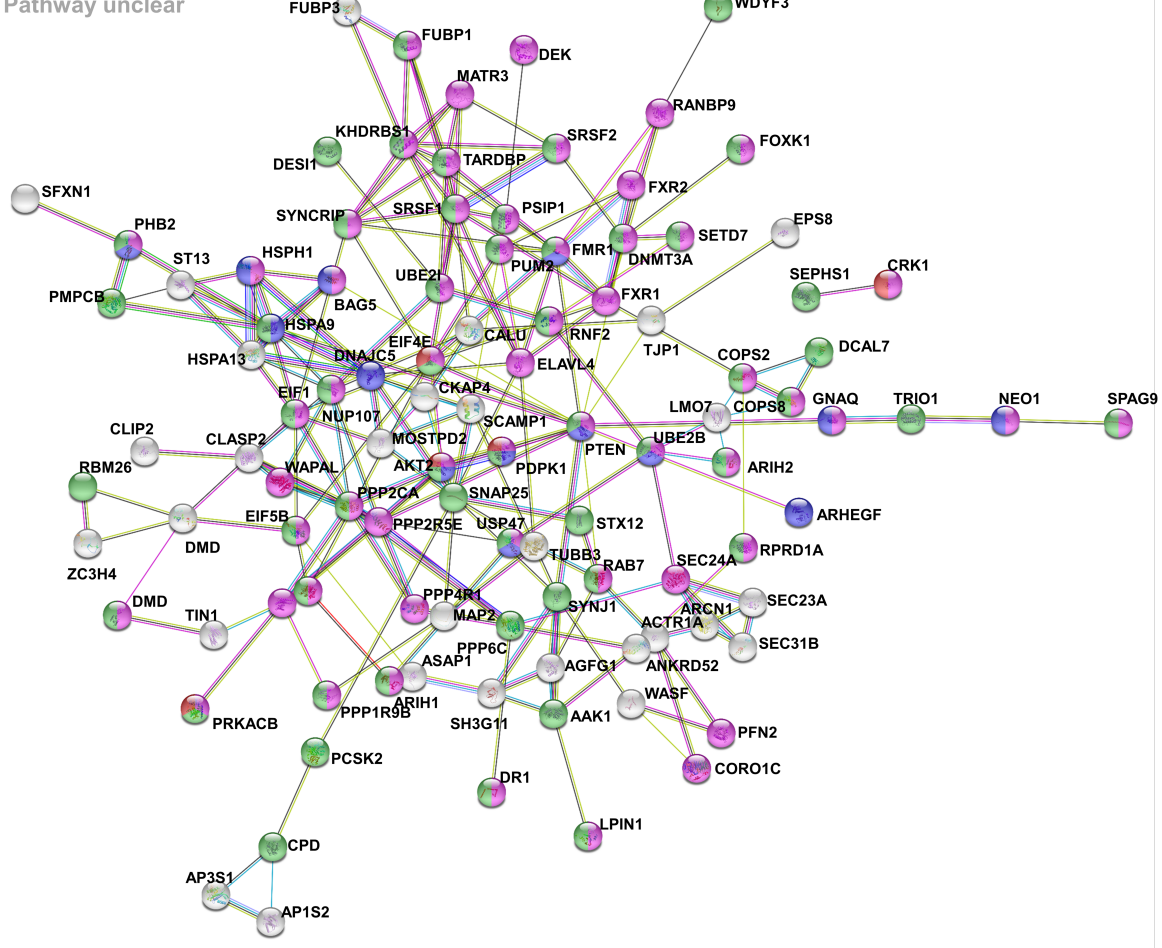

Figure 6. TGP-200c has specific effects on the global MIN6 proteome, traced to the selective inhibition of miR-20oc biogenesis. A) Effect of TGP-2ooc $(2 \mu \mathrm{M})$ on the proteome of MIN6 cells. Proteins encoded by mRNAs that are direct targets of miR-200c, $-200 b$, and -429 (a miR-200 subfamily) are highlighted in blue; direct targets of miR-200a and miR-141 are highlighted in green. Downstream targets were predicted by TargetScan (48). B) On-target effects of TGP-200c observed in global proteomics analysis, grouped by miR-20o subfamilies, 
miR-200c/200b/429 and miR-200a/141 protein. The difference in their downstream targets arises from the single nucleotide difference in their seed regions (U/C in the third position). C) Pathway analysis of 128 proteins predicted to be regulated by miR-200c/-20ob/-429 conducted using STRING analysis (www.string-db.org). Several pathways are selectively modulated by TGP-200, including insulin signaling (red), macromolecule metabolism (pink), general cellular metabolism (green), and negative regulation of cell death (purple) with false discovery rates of $0.025,2.3 \times 10^{-8}, 0.004$, and 0.0009 , respectively. 


\section{References}

1. Colleen M. Connelly, Michelle H. Moon, John S. Schneekloth, Jr., The emerging role of RNA as a therapeutic target for small molecules. Cell Chem. Biol. 23, 1077-1090 (2016).

2. Y. Tor, Targeting RNA with small molecules. Chembiochem 4, 998-1007 (2003).

3. A. Donlic, A. E. Hargrove, Targeting RNA in mammalian systems with small molecules. WIREs RNA 9, e1477 (2018).

4. A. J. Angelbello et al., Using genome sequence to enable the design of medicines and chemical probes. Chem. Rev. 118, 1599-1663 (2018).

5. M. G. Costales et al., Small molecule inhibition of microRNA-210 reprograms an oncogenic hypoxic circuit. J. Am. Chem. Soc. 139, 3446-3455 (2017).

6. M. Ha, V. N. Kim, Regulation of microRNA biogenesis. Nat. Rev. Mol. Cell. Biol. 15, 509-524 (2014).

7. D. P. Bartel, MicroRNAs: target recognition and regulatory functions. Cell 136, 215-233 (2009).

8. J. Krutzfeldt et al., Silencing of microRNAs in vivo with 'antagomirs'. Nature 438, 685-689 (2005).

9. D. D. Vo, M. Duca, Design of multimodal small molecules targeting miRNAs biogenesis: synthesis and in vitro evaluation. Methods Mol. Biol. 1517, 137-154 (2017).

10. D. D. Vo et al., Building of neomycin-nucleobase-amino acid conjugates for the inhibition of oncogenic miRNAs biogenesis. Org. Biomol. Chem. 16, 6262-6274 (2018).

11. S. P. Velagapudi, S. M. Gallo, M. D. Disney, Sequence-based design of bioactive small molecules that target precursor microRNAs. Nat. Chem. Biol. 10, 291-297 (2014).

12. M. G. Costales, Y. Matsumoto, S. P. Velagapudi, M. D. Disney, Small molecule targeted recruitment of a nuclease to RNA. J. Am. Chem. Soc. 140, 6741-6744 (2018).

13. M. G. Costales et al., Small-molecule targeted recruitment of a nuclease to cleave an oncogenic RNA in a mouse model of metastatic cancer. Proc. Natl. Acad. Sci. U. S. A. 117, 2406-2411 (2020).

14. X. Liu et al., Targeted degradation of the oncogenic microRNA 17-92 cluster by structure-targeting ligands. J. Am. Chem. Soc. 142, 6970-6982 (2020).

15. Y. Altuvia et al., Clustering and conservation patterns of human microRNAs. Nucleic Acids Res. 33, 2697-2706 (2005).

16. S. P. Kabekkodu et al., Clustered miRNAs and their role in biological functions and diseases. Biol. Rev. Camb. Philos. Soc. 93, 1955-1986 (2018).

17. S. R. Filios et al., MicroRNA-200 is induced by thioredoxin-interacting protein and regulates Zeb1 protein signaling and beta cell apoptosis. J. Biol. Chem. 289, 36275-36283 (2014).

18. B. F. Belgardt et al., The microRNA-200 family regulates pancreatic beta cell survival in type 2 diabetes. Nat. Med. 21, 619-627 (2015).

19. S. Griffiths-Jones, H. K. Saini, S. van Dongen, A. J. Enright, miRBase: tools for microRNA genomics. Nucleic Acids Res. 36, D154-158 (2008).

20. A. Kozomara, S. Griffiths-Jones, miRBase: integrating microRNA annotation and deep-sequencing data. Nucleic Acids Res. 39, D152-157 (2011).

21. D. P. Bartel, MicroRNAs: genomics, biogenesis, mechanism, and function. Cell 116, 281-297 (2004).

22. S. P. Velagapudi, S. M. Gallo, M. D. Disney, Sequence-based design of bioactive small molecules that target precursor microRNAs. Nat. Chem. Biol. 10, 291-297 (2014).

23. M. D. Disney et al., Inforna 2.0: a platform for the sequence-based design of small molecules targeting structured RNAs. ACS Chem. Biol. 11, 1720-1728 (2016).

24. A. Pushechnikov et al., Rational design of ligands targeting triplet repeating transcripts that cause RNA dominant disease: application to myotonic muscular dystrophy type 1 and spinocerebellar ataxia type 3. J. Am. Chem. Soc. 131, 9767-9779 (2009). 
25. H. S. Haniff, A. Graves, M. D. Disney, Selective small molecule recognition of RNA base pairs. ACS Comb. Sci. 20, 482-491 (2018).

26. H. C. Kolb, M. G. Finn, K. B. Sharpless, Click chemistry: diverse chemical function from a few good reactions. Angew. Chem. Int. Ed. Engl. 40, 2004-2021 (2001).

27. M. D. Disney, Targeting RNA with small molecules to capture opportunities at the intersection of chemistry, biology, and medicine. J. Am. Chem. Soc. 141, 6776-6790 (2019).

28. L. Elghazi, E. Bernal-Mizrachi, Akt and PTEN: $\beta$-cell mass and pancreas plasticity. Trend. Endocrinol. Metab. 20, 243-251 (2009).

29. S. A. Mortimer, M. A. Kidwell, J. A. Doudna, Insights into RNA structure and function from genomewide studies. Nat. Rev. Genet. 15, 469-479 (2014).

30. M. Lindow et al., Assessing unintended hybridization-induced biological effects of oligonucleotides. Nat. Biotechnol. 30, 920-923 (2012).

31. L. Stojic et al., Specificity of RNAi, LNA and CRISPRi as loss-of-function methods in transcriptional analysis. Nucleic Acids Res. 46, 5950-5966 (2018).

32. T. Yoshida et al., Estimated number of off-target candidate sites for antisense oligonucleotides in human mRNA sequences. Genes to Cells 23, 448-455 (2018).

33. S. G. Rzuczek et al., Precise small-molecule recognition of a toxic CUG RNA repeat expansion. Nat. Chem. Biol. 13, 188-193 (2017).

34. A. J. Angelbello et al., Precise small-molecule cleavage of an $r(C U G)$ repeat expansion in a myotonic dystrophy mouse model. Proc. Natl. Acad. Sci. U. S. A. 116, 7799-7804 (2019).

35. M. G. Costales, S. G. Rzuczek, M. D. Disney, Comparison of small molecules and oligonucleotides that target a toxic, non-coding RNA. Bioorg. Med. Chem. Lett. 26, 2605-2609 (2016).

36. R. I. Benhamou et al., Structure-specific cleavage of an RNA repeat expansion with a dimeric small molecule is advantageous over sequence-specific recognition by an oligonucleotide. ACS Chem. Biol. 15, 485-493 (2020).

37. A. J. Angelbello, M. E. DeFeo, C. M. Glinkerman, D. L. Boger, M. D. Disney, Precise targeted cleavage of a $r(C U G)$ repeat expansion in cells by using a small-molecule-deglycobleomycin conjugate. ACS Chem. Biol. 15, 849-855 (2020).

38. B. Humphries, C. Yang, The microRNA-200 family: small molecules with novel roles in cancer development, progression and therapy. Oncotarget 6, 6472-6498 (2015).

39. N. Hill et al., Glomerular endothelial derived vesicles mediate podocyte dysfunction: A potential role for miRNA. PloS one 15, e0224852 (2020).

40. J. F. Arambula, S. R. Ramisetty, A. M. Baranger, S. C. Zimmerman, A simple ligand that selectively targets CUG trinucleotide repeats and inhibits MBNL protein binding. Proc. Nat. Acad. Sci. U. S. A. 106, 16068-16073 (2009).

41. P. Tiwari, Recent trends in therapeutic approaches for diabetes management: A comprehensive update. J. Diabetes Res. 2015, 340838-340838 (2015).

42. S. P. Velagapudi, A. Pushechnikov, L. P. Labuda, J. M. French, M. D. Disney, Probing a 2aminobenzimidazole library for binding to RNA internal loops via two-dimensional combinatorial screening. ACS Chem. Biol. 7, 1902-1909 (2012).

43. N. Peyret, P. A. Seneviratne, H. T. Allawi, J. SantaLucia, Jr., Nearest-neighbor thermodynamics and NMR of DNA sequences with internal A.A, C.C, G.G, and T.T mismatches. Biochemistry 38, 34683477 (1999).

44. J. D. Puglisi, I. Tinoco, Jr., Absorbance melting curves of RNA. Method. Enzymol. 180, 304-325 (1989).

45. S. P. Velagapudi et al., Defining RNA-small molecule affinity landscapes enables design of a small molecule inhibitor of an oncogenic noncoding RNA. ACS Cent. Sci. 3, 205-216 (2017). 
46. X. Rao, X. Huang, Z. Zhou, X. Lin, An improvement of the $2^{\wedge}$ (-delta delta CT) method for quantitative real-time polymerase chain reaction data analysis. Biostat. Bioinforma. Biomath. 3, 71-85 (2013).

47. J. Cox, M. Mann, MaxQuant enables high peptide identification rates, individualized p.p.b.-range mass accuracies and proteome-wide protein quantification. Nat. Biotechnol. 26, 1367 (2008).

48. V. Agarwal, G. W. Bell, J.-W. Nam, D. P. Bartel, Predicting effective microRNA target sites in mammalian mRNAs. elife 4, e05005 (2015). 